\title{
Patterns Of Torus Mandibularis And Torus Palatinus Among Dental Patients Of A Public Hospital In Karachi
}

Ramsha Azhar, Salik Rasool, Bushra Jabeen, Umer Hasan, Hasan Baber, Bilal Hussain, Mansoor Akhtar

- - - - - - - - - - - - - - - - - - - - - - - - - - -

ABSTRACT

Objective: To evaluate the patterns of Torus mandibularis (TM) and Torus palatinus (TP) among the patients attending the dental department of a teaching hospital in Karachi focusing on the age and gender.

Study Design and Setting: A cross-sectional study conducted at dental OPD of Dow International Dental College/Hospital DUHS, conducted from November 2017 to October 2018.

Methodology: The calculated sample size of the study was 278. Written consent was taken from each participant prior to starting investigation. The questionnaire comprises of 17 variables, evaluating demographics, state of dentition and characteristics of presence of any suspected tori. Participants were examined via sterilized mouth mirror and probe. Impressions were then taken from the participants having positive findings to further evaluate the size and shape to tori.

Results: The prevalence of (TM) and (TP) were found to be $3.3 \%$ and $0.6 \%$ respectively. (TM) was predominantly found in males and (TP) was more frequent in females. Both (TM) and (TP) were most prevalent during fifth decade of life irrespective of gender. Bilateral unilobulated (TM) in the premolar region of the mandible were found to be most recurrent. Lobulated (TP) of large size was found to be prevalent in this study.

Conclusion: Study has shown very less prevalence of tori in population of Karachi, Pakistan. The prevalent patterns of tori were found to be bilateral unilobulated in the premolar region of the mandible and large, lobular in the palate. The relationship of tori with age and gender was not significant.

Keywords: Patterns, Prevalence, Torus mandibularis, Torus Palatinus,

\section{INTRODUCTION:}

Tori and exostoses are bony protuberances, composed of dense compact bone with minimal amount of bone marrow and are usually localized. ${ }^{1}$ These benign, bony outgrowths are designated according to the anatomic location. ${ }^{2,3}$ When they develop at the midline of the palate, they are referred as torus palatinus and when found at the lingual aspect of

ГRamsha Azhar, -

I Lecturer, Department of Oral Pathology,

I Dow International Dental College, DU

I

Salik Rasool

Associate Professor, Department of Oral Pathology,

I Dow International Dental College, DUHS

I Bushra Jabeen

I Associate Professor, Department of Prosthodontics,

I Dow International Dental College, DUHS

I Umer Hasan

Associate Professor, Department of Oral Medicine,

1

Hasan Baber

Assistant Professor, Department of Oral Biology,

I Dow International Dental College, DUHS

I Bilal Hussain

I Lecturer, Department of Oral Pathology,

I Dow International Dental College, DUHS

I Mansoor Akhtar

Lecturer, Department of Prosthodontics,

Dow International Dental College, DUHS

Received: 29-11-2018

Accepted: 23-04-2019 the mandible, they are attributed as torus mandibularis ${ }^{1,2}$ They continue to grow gradually during second and third decades of life and are usually unrecognized until middle age. $^{3}$

According to the shape, Torus Palatinus (TP) is categorized as flat, spindle-shaped, nodular and lobular while Torus mandibularis (TM) is classified as unilateral or bilateral solitary, unilateral or bilateral multiple and bilateral combined. ${ }^{1,3}$ With respect to size,(TP) can be grouped as small (less than $3 \mathrm{~mm})$, medium (3-6mm) and large (more than $6 \mathrm{~mm}){ }^{1}(\mathrm{TM})$ may display modifications in size, but they are generally small. ${ }^{1}$ Most commonly observed (TP) is flat shaped whereas most commonly found (TM) is bilateral solitary type. ${ }^{7}$ They are diagnosed by clinical and radiographic examination.

The exact etiology of tori is unknown, but researches have shown that its occurrence is multifactorial; including genetic and environmental factors. ${ }^{1,2}$ The threshold theory by Gorsky et al is widely accepted. According to this theory, autosomnal dominant genetic trait is primarily required, but environmental factors must reach threshold levels for the development of tori. ${ }^{2}$ Other predisposing factors include trauma, drugs, infection, discontinued growth, masticatory stress, dietary habits and nutritional imbalances. ${ }^{1,2}$

Various studies have reported prevalence of tori to vary widely globally, ranging from 0.4 to $61.7 \%$ and 1 to $64 \%$ for (TP) and (TM) respectively. ${ }^{2}$ Tori are predominantly observed in patients with more than 40 years of age. ${ }^{2}$ 
After a comprehensive search on Medline and Cochrane library, it was observed that there is lack of studies in Sindh, Pakistan regarding prevalence and pattern of tori, with one study in Karachi reporting $8.6 \%$ and $3.7 \%$ prevalence of (TM) and (TP) respectively among patients attending private dental hospital.

The Objective of current study was To evaluate the patterns of Torus mandibularis (TM) and Torus palatinus (TP) among the patients attending the dental department of a teaching hospital in Karachi focusing on the age and gender.

\section{METHODOLOGY:}

A cross-sectional study was conducted at Dow International Dental College/Hospital (Chanesar and Ojha campus) DUHS. The study was conducted from November 2017 to October 2018 (Duration of 1 year). Participants of the study included patients attending dental OPD of DIDC, DUHS for different dental problems.

Sample size was calculated by using online sample size calculator Openepi (proportion based), keeping 5\% standard mean error and $95 \%$ confidence limit. The calculated sample size of the study was 278 . This sample size was similar to the literature available of other studies that were conducted on tori.

Data was collected by trained dental surgeons and post graduates for this study. Initially, a pilot study comprising of 25 participants (which were not included in the main study) was conducted to determine internal reliability of the questionnaire via Cronbach's alpha internal reliability test using SPSS version 25. Three variables related to the occupation, number of working hours and working environment were removed from the questionnaire because these questions were irrelevant to the study and by removing these questions the Cronbach's alpha test value was found to be increased. Questionnaire was validated via face validity by experts in the subjects (Oral pathologist and Research analyst).

Limitations of the present study included males and females of all age groups ranging from 10 to 80 years and of all ethnic background.

Written consent was taken from each participant prior to starting investigation. The subjects who did not give consent were excluded from the research. Each participant was interviewed by a trained field investigator via aid of custommade questionnaire. The questionnaire comprises of 17 variables, evaluating demographics, state of dentition and characteristics of presence of any suspected tori. The first five questions were related to sociodemographic details (age, gender, ethnic background) of the patient while the remaining questions are related to presence or absence of tori. The presence of any suspected (TM) and (TP) was done by visual inspection and palpation. Participants were examined via sterilized mouth mirror and probe. Impressions were then taken from the participants having positive findings to further evaluate the size and shape to tori. If presence of (TM) is observed, then its location (anterior, middle and posterior), shape (unilobulated, bilobulated and multilobulated) in the mandible are recorded and if (TP) is observed, then its size (small, medium and large) and shape (flat, spindle-shaped, nodular and lobular) are recorded in the questionnaire.

Total of two hundred and eighty (280) questionnaires were filled. Ten questionnaires were excluded from the research due to incomplete information.

For interpretation of data SPSS version 25 (Chicago, IL, USA) was used. To determine presence of tori with respect to age and gender, Chi Square test was applied. To determine any possible correlation between Independent Variable (Gender and Age) with Dependent Variable (Tori), Pearson correlation was utilized and to evaluate probability of tori occurring with change in age and gender logistic regression was used.

\section{RESULTS:}

A total of 270 subjects, including 135 males (50\%) and 135 females $(50 \%)$ were examined.10 participants had positive findings for tori. The overall prevalence of (TM) was found to be $3.3 \%$ ( 9 subjects) whereas (TP) was found to be $0.6 \%$ (1 subject).

Table 1 represents the occurrence of (TM) in relation to age. (TM) was reported in $9(3.3 \%)$ out of 270 subjects. The highest frequency was found in the 40-49year age group where $3(1.1 \%)$ subjects had positive findings out of 104 subjects, while the lowest frequency was found in 10-19year age group where no subjects had positive findings out of the 10 subjects examined.(TP)was recorded in $1(0.4 \%)$ out of 270 subjects. The only case of (TP) recorded was found in the 41-50year age group. No significant relationship was found between occurrence of (TM) and (TP) and age $(\mathrm{P}>0.05)$.

Table 1 also summarizes the occurrence of (TM) and (TP) in relation to gender. Out of the 9 subjects with (TM), $5(1.9 \%)$ cases reported were found in males while $4(1.5 \%)$ cases reported were found in females. The only case reported of (TP) was found in a female subject. No significant relation is seen between occurrence of tori and gender $(\mathrm{P}>0.05)$.

Table 2 summarizes the occurrence of (TM) and (TP) in relation to ethnic background. The highest frequency of (TM) was found in Urdu speaking subjects where 5(1.9\%) out of $9(3.3 \%)$ cases were observed. Occurrence in (TM) in other ethnic backgrounds were found to be $3(1.1 \%)$ out of 9 in Punjabi subjects and $1(0.3 \%)$ out of 9 in Pathan subjects. The only case of (TP) was found in subjects with Punjabi ethnic background.

Table 3 summarizes the location of (TM) on the mandibular lingual aspect in 270 subjects. The highest incidence of 
Pattern And Frequency Of Torus Mandibularis And Torus Palatinus Among Dental Patients Of A Public Hospital In Karachi

(TM) was found in the middle (premolar) area where $7(2.6 \%)$ out of $9(3.3 \%)$ cases were observed on the right side and $6(2.2 \%)$ out of $7(2.6 \%)$ cases were observed on the left side of the mandible. $2(0.6 \%)$ cases were observed in the anterior mandibular region on the right side whereas only $1(0.4 \%)$ case was observed on the left side of the mandible. The least incidence of (TM) was found in posterior mandibular region where no cases were observed in both left and right sides of the mandible.

Table 3 also summarizes the distribution of (TM) according to shape. The most common shape was bilateral unilobulated (TM). $7(2.6 \%)$ out of $9(3.3 \%)$ cases were detected of such type. In $2(0.6 \%)$ out of 9 cases, unilateral unilobulated (TM) was found. No cases of unilateral or bilateral bilobulated and multilobulated (TM) were observed.

Table 4 presents the shape and size of (TP) on the hard

\begin{tabular}{|c|c|c|c|c|c|c|c|c|c|c|}
\hline & & \multicolumn{6}{|c|}{ AGE DISTRIBUTION } & \multicolumn{3}{|c|}{$\begin{array}{c}\text { GENDER } \\
\text { DISTRIBUTION }\end{array}$} \\
\hline & & $\begin{array}{l}10 y r s- \\
20 y r s\end{array}$ & $\begin{array}{l}21 \text { yrs- } \\
30 y r s\end{array}$ & $\begin{array}{l}31 y r s- \\
40 y r s\end{array}$ & \begin{tabular}{|l|}
$41 \mathrm{yrs}-$ \\
$50 \mathrm{yrs}$
\end{tabular} & \begin{tabular}{|l|}
$51 y r s-$ \\
$80 y r s$
\end{tabular} & Total & Male & Female & Total \\
\hline Torus & Present & 0 & 0 & 0 & 1 & 0 & 1 & 0 & 1 & 1 \\
\hline Palatinus & Absent & 10 & 23 & 60 & 103 & 73 & 269 & 135 & 134 & 269 \\
\hline Total & & 10 & 23 & 60 & 104 & 73 & 270 & 135 & 135 & 270 \\
\hline Torus & Present & 0 & 2 & 2 & 3 & 2 & 9 & 5 & 4 & 9 \\
\hline Mandibularis & Absent & 10 & 21 & 58 & 101 & 71 & 261 & 130 & 131 & 261 \\
\hline Total & & 10 & 23 & 60 & 104 & 73 & 270 & 135 & 135 & 270 \\
\hline
\end{tabular}

TABLE 1: Presents the age and gender distribution of subjects in relation to presence of TM and TP. TM was more frequent in males ( 5 out of 9 subjects). TP was found in one female subject. TM and TP were most common in 40-49yrs of age ( 4 out of 10 subjects)

\begin{tabular}{|l|c|c|c|c|c|c|c|c|}
\hline & \multicolumn{7}{|c|}{ Ethnic Background } \\
\hline & Urdu speaking & Sindhi & Punjabi & Balochi & Pathan & Others & Total \\
\hline Torus Mandibularis & 5 & 0 & 3 & 0 & 1 & 0 & $\mathbf{9}$ \\
\hline Torus Palatinus & 0 & 0 & 1 & 0 & 0 & 0 & $\mathbf{1}$ \\
\hline
\end{tabular}

Table 2: Presents the ethnic background distribution of subjects in relation to presence of TM and TP. TM was found to be most common in Urdu speaking subjects (5 out of 9) whereas TP was found in Punjabi subjects.

\begin{tabular}{|c|c|c|c|c|c|c|c|c|c|}
\hline & & Anterior & Middle & Posterior & Total & $\begin{array}{c}\text { Uni- } \\
\text { Lobulated }\end{array}$ & $\begin{array}{c}\text { Bi- } \\
\text { Lobulated }\end{array}$ & $\begin{array}{c}\text { Multi- } \\
\text { Lobulated }\end{array}$ & Total \\
\hline \multirow{2}{*}{$\begin{array}{l}\text { Torus } \\
\text { mandibularis }\end{array}$} & RIGHT SIDE & 2 & 7 & 0 & 9 & 9 & 0 & 0 & 9 \\
\hline & LEFT SIDE & 1 & 6 & 0 & 7 & 7 & 0 & 0 & 7 \\
\hline
\end{tabular}

TABLE 3: Summarizes the distribution of TM in relation to location and shape. Most common location of TM was found in middle [premolar region] ( 7 out of 9 at the right side of the mandible), anterior region ( 2 out of 9 at the right side of the mandible). Most frequent shape of TM was unilobulated ( 9 out of 9 at the right and 7 out of 7 at the left side of mandible).

\begin{tabular}{|c|c|c|c|c|c|c|c|c|c|}
\hline \multicolumn{4}{|c|}{ TP SIZE } & \multicolumn{5}{c|}{ TP SHAPE } & \\
& $\begin{array}{c}\text { Small } \\
(<3 \mathrm{~mm})\end{array}$ & $\begin{array}{c}\text { Medium } \\
(3-6 \mathrm{~mm})\end{array}$ & $\begin{array}{c}\text { Large } \\
(>6 \mathrm{~mm})\end{array}$ & Total & Flat & $\begin{array}{c}\text { Spindle- } \\
\text { shaped }\end{array}$ & Nodular & Lobular & Total \\
\hline Torus palatinus & 0 & 0 & 1 & $\mathbf{1}$ & 0 & 0 & 0 & 1 & $\mathbf{1}$ \\
\hline
\end{tabular}

TABLE 4: Summarizes the distribution of shape and size of TP. The only TP found was lobular in shape and large $(>6 \mathrm{~mm})$ in size. 
palate. The most frequent shape of (TP) was found to be lobular. No cases of flat, nodular or spindle-shaped (TP) were found. The most frequent size of (TP) was found to be large ( $>6 \mathrm{~mm})$. No cases of small or medium sized (TP) were found in this study.

\section{DISCUSSION:}

Prevalence rates of tori have been studied in various populations of the world. Epidemiological studies report that in Turkish population, the prevalence of (TP) is between 21- $45 \% .{ }^{3}$ There is female predilection observed. ${ }^{3}$ According to a study conducted in Northern Malaysia prevalence rates of (TP) and (TM) were found to be $12 \%$ and $2.8 \%$ respectively. ${ }^{2}$ Epidemiological studies in India report that prevalence of (TP) and (TM) is $1.3 \%$ and $6.9 \%$ respectively. ${ }^{7}$ Mirza et al from Karachi, showed prevalence rates of (TM) to be $8.6 \%$ and of (TP) to be $3.7 \%{ }^{8}{ }^{811}$ In the present study, the prevalence rates of (TM) and (TP) were found to be $3.3 \%$ and $0.6 \%$ respectively. The results showed decreased prevalence as compared to other studies, but this may due to decreased sample size.

The current study also investigates the occurrence of (TM) and (TP) in relation to gender. (TP) is reported to be more frequent in females as compared to males, and this was accredited to the dominant $\mathrm{X}$ chromosome. ${ }^{1,12}$ The current study displayed similar results with high prevalence in female subjects which is in line with the studies conducted by Sathya et al in Northern Malaysia, Sisman et al in Turkey and Mirza et al in Karachi, Pakistan., ${ }^{2,11}$ TM is found to be more common in males ${ }^{1,13,14,18}$ which is similar to the results of this study. However, contrasting results have been seen in study conducted by Sathya et al and Mirza et al, showing female predilection., ${ }^{2}, 11$

Tori tend to increase with age and become most prominent during the middle phase of life. ${ }^{2,15}$ In the present study, the prevalence of tori is seen to be most frequent in the fifth decade (40-49 years of age) which is similar to the previous researches conducted. ${ }^{2,8,11,15,16,17}$ The least incidence was found during the second (10-19 years of age). The increase in size of tori with age was suggested by Ludvig K. Haugen. According to his study, the masticatory stress is increased from adolescence to adulthood and decreased during senescence. ${ }^{2,18}$ Further research conducted by Sonnier et al suggested that the regression of tori after 60 years of age was due to decreased masticatory function as a result of edentulism. ${ }^{2,19}$

The present study investigated the relationship of the various ethnic backgrounds with presence of (TM) and (TP).

The results showed that (TM) was most prevalent in Urdu speaking population and least prevalent in Balochi and Sindhi population which is similar to local literature reported by Mirza et al. ${ }^{8}$ However, this result does not provide the true representation of all ethnicities due to limited sample size of the study.
The occurrence of (TM) was also investigated in relation to the location of tori. In our study, majority of (TM) were observed to be bilaterally symmetrical which are similar to previous studies conducted by Mirza et al in Karachi and Hiremath et al in Malay population. ${ }^{2,8,20,21}$ The most frequent location of (TM) was observed in premolar region. Unilateral (TM) was observed more commonly on right side of the mandible as compared to the left side. These findings are in congruence with past researches conducted. ${ }^{8,22}$

The distribution of (TP) according to shape and size were also investigated. In the current study, the most common pattern of (TP) observed was large in size and lobular in shape. These results were found to be in contrast with Sathya et al and Gorsky et al where smooth variety of (TP) was found to be predominant. ${ }^{2,}{ }^{23}$ A local study in Karachi conducted by Mirza et al reported spindle shaped and medium sized (TP) to be most common. ${ }^{11}$ The contrasting results found could be due to decreased sample size as compared to other studies conducted.

The exact etiology of tori is still unclear, but the role of environmental and genetic factors makes its etiology multifactorial. The environmental factors including dietary habits and occlusal stresses could play a major role in the development of tori. ${ }^{2}$ The association of tori with medical conditions or syndromes, dental anomalies or excessive drug usage was not observed in the present study. A study conducted by Sasaki reported relationship between chronic phenytoin therapy with presence of tori. ${ }^{27}$ Another study by Morrison et al reported significant association between tori and temporomandibular dysfunction and tooth attrition. ${ }^{28}$

Presence of tori necessitates the need to redesign denture prosthesis and may require surgical removal in rare cases. A new technique was introduced by Rocca and colleagues for surgical removal of tori. ${ }^{8,}{ }^{26}$ In order to facilitate easy removal of tori and to avoid damage to the surrounding tissues, Er: YAG laser was utilized by them. Healing process has also been seen to be enhanced by this technique. ${ }^{8,26}$ Furthermore, studies with larger sample size should be conducted to conclude the actual prevalence of tori in Pakistani population. Future studies can deduce the relation of drug usage, dental anomalies and systemic diseases with tori.

\section{CONCLUSION:}

Study has shown very less prevalence of tori in population of Karachi, Pakistan. The prevalent patterns of tori were found to be bilateral unilobulated in the premolar region of the mandible and large, lobular in the palate. The relationship of tori with age and gender was not significant.

\section{REFERENCES:}

1. AlZarea, B. K. (2016). Prevalence and pattern of torus palatinus and torus mandibularis among edentulous patients of Saudi Arabia. Clinical interventions in aging, 2016;11: 209. 
2. Sathya, K., Kanneppady, S. K., \&Arishiya, T. (2012). Prevalence and clinical characteristics of oral tori among outpatients in Northern Malaysia. Journal of oral biology and craniofacial research, 2012; 2(1): 15-19.

3. Sisman, Y., Ertas, E. T., Gokce, C., \&Akgunlu, F. (2008). Prevalence of torus palatinus in cappadocia region population of Turkey. European journal of dentistry, 2008;2:269.

4. Singh, A. K., Ramachandra, S. S., Arora, S., Dicksit, D. D., Kalyan, C. G., \& Singh, P. Prevalence of oral tori and exostosis in Malaysian population-A cross-sectional study. Journal of oral biology and craniofacial research. 2017; 7(3): 158-160.

5. Morita, K., Tsuka, H., Shintani, T., Yoshida, M., Kurihara, H., \&Tsuga, K. Prevalence of torus mandibularis in young healthy dentate adults. Journal of Oral and Maxillofacial Surgery,2017; 75(12: 2593-98.

6. Uysal, S., Çaðýrankaya, B. L., \&Hatipoðlu, M. G. Do gender and torus mandibularis affect mandibular cortical index? A cross-sectional study. Head \& face medicine, 2007;3(1):37.

7. Patil, S., Maheshwari, S., \& Khandelwal, S. K. Prevalence of torus palatinus and torus mandibularis in an Indian population. Saudi Journal of Oral Sciences, 2014;1(2): 94.

8. Mirza, D., Khokhar, N. H., Katpar, S. J., Khan, Y., Hakeem, S., \&Musani, M. A. Prevalence of torus mandibularis among various ethnic groups in Karachi: A cross sectional study. Pak Oral Dent J, 2013; 33: 277-80.

9. Qazi, S. S., \& BDS, A. A. A. Prevalence of torus palatinus among 300 Indonesian patients. Pakistan Oral and Dental Journal,2013; 27(1): 89-91.

10. Abdul-Ameer, F. M. Correlation of Prevalence of Torus Palatinus and Torus Mandibularis with the Form of Maxillary and Mandibular Arches in Iraqi Population. Journal of Medical and Dental Science Research, 2016; 3(6): 14-22.

11. Mirza, D., Hakeem, S., Omer, S. A., \& Rizvi, K. F. Frequency Of Torus Palatinus; A Study Done In A Teaching Hospital In Karachi. Pakistan Oral \& Dental Journal, 2013;33(1).

12. García-García, A. S., Martínez-González, J. M., Gómez-Font, R., Soto-Rivadeneira, Á., \& Oviedo-Roldán, L. (2010). Current status of the torus palatinus and torus mandibularis. Med Oral Patol Oral Cir Bucal, 2010;15(2): e353-e360.

13. Chew, C. L., \& Tan, P. H. Torus palatinus. A clinical study. Australian dental journal, 1984;29(4): 245-248.

14. Jainkittivong, A., Apinhasmit, W., \& Swasdison, S. Prevalence and clinical characteristics of oral tori in 1,520 Chulalongkorn University Dental School patients. Surgical and Radiologic Anatomy, 2007;29(2): 125-131.

15. Jainkittivong, A., \&Langlais, R. P. Buccal and palatal exostoses: prevalence and concurrence with tori. Oral Surgery, Oral Medicine, Oral Pathology, Oral Radiology, and Endodontology, 2000; 90(1): 48-53.
16. Eggen, S., Natvig, B., \& Gåsemyr, J. Variation in torus palatinus prevalence in Norway. European Journal of Oral Sciences, 1994;102(1): 54-59.

17. KraljeviæŠimunkoviæ, S., Božiæ, M., Z Alajbeg, I., Dulèiæ, N., \&Vuèiæeviæ Boras, V. (2011). Prevalence of torus palatinus and torus mandibularis in the Split-Dalmatian County, Croatia. Collegium antropologicum, 2011;35(3): 637-41.

18. Haugen, L. K. Palatine and mandibular tori: a morphologic study in the current Norwegian population. Acta Odontologica Scandinavica, 1992;50(2): 65-77.

19. Sonnier, K. E., Horning, G. M., \& Cohen, M. E. Palatal tubercles, palatal tori, and mandibular tori: prevalence and anatomical features in a US population. Journal of periodontology, 1999; 70(3): 329-36.

20. Hiremath, V. K., Husein, A., \& Mishra, N. Prevalence of torus palatinus and torus mandibularis among Malay population. Journal of International Society of Preventive \& Community Dentistry, 2011;1(2): 60.

21. Ihunwo, A. O., \& Phukubye, P. The frequency and anatomical features of torus mandibularis in a Black South African population. HOMO-Journal of Comparative Human Biology, 2006;57(4): 253-262.

22. Apinhasmit, W., Jainkittivong, A., \&Swasdison, S. Torus palatinus and torus mandibularis in a Thai population. Sci Asia, 2002;28: 105-11.

23. Gorsky, M., Raviv, M., Kfir, E., \&Moskona, D. Prevalence of torus palatinus in a population of young and adult Israelis. Archives of oral biology, 1996;41(6): 623-625.

24. Sasaki H, Ikedo D, Kataoka M, Kido J, Kitamura S, Nagata T.Pronounced palatal and mandibular tori observed in a patient with chronic phenytoin therapy: a case report. J Periodontol.1999; 70(4): 445-8.

25. Al Quran FA, Al-Dwairi ZN. Torus palatinus and torus mandibularis in edentulous patients. J Contemp Dent Pract. 2006; 7(2): 112-9.

26. Rocca JP, Raybaud H, Merigo E, Vescovi P, Fornaini C. Er: YAG Laser: A New Technical Approach to Remove Torus Palatinus and Torus Mandibularis. Case Rep Dent. 2012;2012:487802. doi: 10.1155/2012/487802.

27. García-García AS, Martínez-González JM, Gómez-Font R, Soto-Rivadeneira Á, Oviedo-Roldán L. Current status of the torus palatinus and torus mandibularis. Med Oral Patol Oral Cir Bucal. 2010;15(2):e353-60.

28. Morrison MD, Tamimi F. Oral tori are associated with local mechanical and systemic factors: a case-control study. Journal of Oral and Maxillofacial Surgery. 2013;71(1):14-22. 